\title{
Clinical and molecular features of innate and acquired resistance to anti-PD-1/PD-L1 therapy in lung cancer
}

\author{
Shalin Shah ${ }^{1, *}$, Kevin Wood ${ }^{2, *}$, Brian Labadie ${ }^{2}$, Brian Won ${ }^{2}$, Ryan Brisson ${ }^{2}$, Theodore \\ Karrison ${ }^{3}$, Thomas Hensing ${ }^{2}$, Mark Kozloff', Riyue Bao ${ }^{4}$, Jyoti D. Patel ${ }^{2}$ and Jason J. \\ Luke $^{2}$ \\ ${ }^{1}$ Department of Medicine, NorthShore University HealthSystems, Chicago, IL, USA \\ ${ }^{2}$ Department of Medicine, University of Chicago, Chicago, IL, USA \\ ${ }^{3}$ Department of Public Health Sciences, University of Chicago, Chicago IL, USA \\ ${ }^{4}$ Center for Research Informatics and Department of Pediatrics, University of Chicago, Chicago, IL, USA \\ *These authors contributed equally to this work \\ Correspondence to: Jason J. Luke, email: jluke@medicine.bsd.uchicago.edu
}

Keywords: non-small cell lung cancer; anti-PD-1/PD-L1 therapy; acquired resistance; immunotherapy; innate resistance Received: September 04, $2017 \quad$ Accepted: December 08, $2017 \quad$ Published: December 15, 2017

Copyright: Shah et al. This is an open-access article distributed under the terms of the Creative Commons Attribution License 3.0 (CC BY 3.0), which permits unrestricted use, distribution, and reproduction in any medium, provided the original author and source are credited.

\section{ABSTRACT}

Hypothesis: The majority of non-small cell lung cancer (NSCLC) patients treated with anti-PD-1/PD-L1 therapy develop either innate or acquired resistance. Across tumor types, the "T cell-inflamed" tumor microenvironment correlates with clinical response to immunotherapy. We hypothesize that clinical characteristics may be predictive of resistance and that "T cell-inflamed" NSCLC tumors can be identified by gene expression profiling.

Results: Of 93 patients, $36(38.7 \%)$ had innate resistance and $57(61.3 \%)$ had initial benefit to immunotherapy. Innate resistance was associated with non-smokers $(p=0.013)$, more involved disease sites $(p=0.011)$, more prior therapy $(p=0.001)$, and a lower albumin level $(p=0.014)$. Among patients with initial benefit, factors associated with subsequent progression-free survival included higher Karnofsky Performance Status (KPS) $(p=\mathbf{0 . 0 0 4})$ and lower depth of response to anti-PD-1 therapy $(p=0.003)$. A "T cell-inflamed" microenvironment was identified in $42 \%$ of TCGA adenocarcinoma samples versus $21.0 \%$ of squamous cell.

Discussion: Specific clinical characteristics appear to be predictive of either innate or acquired resistance to anti-PD-1/PD-L1 therapy. A "T cell-inflamed" tumor was more common in adenocarcinoma than squamous histology.

Methods: A retrospective review of NSCLC patients treated with anti-PD-1/PD-L1 monotherapy. Patients with innate resistance to anti-PD-1/PD-L1 therapy (defined as progression at first CT evaluation) were compared to patients with initial clinical benefit. Among those with initial clinical benefit, we identified prognostic factors for time to progression (acquired resistance) or death. To further corroborate our findings on limited numbers, immune gene expression profiling of all NSCLC samples from the TCGA database was also pursued.

\section{INTRODUCTION}

Lung cancer is the leading cause of cancer-related mortality worldwide [1]. In recent years, treatment for non-small cell lung cancer (NSCLC) has dramatically evolved with the development of the anti-programmed death (PD-1) and anti-programmed death ligand 1 (PD-L1) checkpoint inhibitors. Historically, patients with metastatic disease have been treated with front-line platinum-based chemotherapy, with few efficacious options available 
at time of progression. Phase III studies have now demonstrated improved progression-free (PFS) and overall survival (OS) with PD-L1 inhibitors compared to secondline standard cytotoxic chemotherapy in both squamous and non-squamous NSCLC $[2,3]$. In the front-line setting, for patients with at least $50 \%$ of tumor cells expressing PD-L1, the anti-PD-1 antibody pembrolizumab has demonstrated improvement in progression-free survival (PFS) and overall survival (OS) with fewer adverse events compared to standard chemotherapy [4]. Recently, a randomized phase II trial achieved an objective response when combining pembrolizumab and platinum-doublet chemotherapy compared to chemotherapy alone [5]. Further phase III studies of first-line therapy evaluating monotherapy, combination therapy, and immunotherapy combined with chemotherapy are ongoing.

Despite the success and advancements of checkpoint inhibitors, a majority of patients will experience innate or acquired (initial clinical benefit followed by the development of resistance) resistance. There remains a lack of information regarding predictive clinical and molecular markers of resistance. Most of the information available comes from other tumor types. At the molecular level, expression of PD-L1 in tumor cells and the presence of CD8+ infiltrating T cells within the tumor microenvironment have been associated with a trend towards clinical benefit $[6,7]$. However, these remain as imperfect predictive biomarkers, likely due to the complexity of the relationship between the immune system and the tumor microenvironment. Composite gene expression profiling, demonstrating a more complete picture of the tumor biology, may represent a more optimal biomarker. Data in other tumor types suggests that a "T cell-inflamed" tumor microenvironment identified by immune gene expression studies correlates with an initial response to immunotherapy [8]. This microenvironment is characterized by infiltration of CD8+ $\mathrm{T}$ cells, chemokines, and other innate immune genes and has been found to have prognostic significance for response to immunotherapy $[9,10]$.

Given that PD1/L1 are becoming more prevalent in the treatment of lung cancer, further studies are necessary to identify clinical and molecular predictors of both innate and acquired resistance. In this study, we analyze the clinical characteristics of a cohort of NSCLC patients treated with anti-PD-1/PD-L1 monotherapy. We hypothesized that clinical characteristics would be predictive of either innate resistance, acquired resistance, or long-term benefit. Given the limited archival tissue available in these patient due to the combination of clinical trial participation and/or multiple diagnostic tests, we then chose to analyze the NSCLC cohort of The Cancer Genome Atlas (TCGA) to identify NSCLC subtypes associated with a "T cell-inflamed" microenvironment to further elucidate possible predictive biomarkers.

\section{RESULTS}

\section{Baseline patient characteristics}

A total of 93 NSCLC patients at the three sites were treated with an anti-PD-1/PD-L1 agent and were included in this retrospective study. Surviving patients were followed for a median of 11.5 months. All of the patients were treated off of any investigative protocol, except for two patients treated with the PD-L1 antibody atezolizumab.

Patient baseline characteristics for the entire cohort are described in Table 1. Mean age was 69 years (range 48-87 years). All patients had stage IV disease, with 32 (34.4\%) having M1a disease and 61 (65.6\%) with M1b. A significant proportion of patients, $91.4 \%$, had 3 sites of disease or less. The majority of patients during this study received nivolumab (95.7\%). Similar to phase III trials involving anti-PD-1/PD-L1 therapy, $14.0 \%$ had treated brain metastases prior to initiating therapy.

Of the 93 patients, $n=36$ patients $(38.7 \%)$ had innate resistance and $n=57(61.3 \%)$ had an initial clinical benefit. The median progression-free and overall survival times for the entire cohort were 5.4 and 11.0 months.

\section{Initial resistance characteristics}

Compared to patients with an initial benefit, those with innate resistance were more likely to be non-smokers $(30 / 36, p=0.013)$ and smoked fewer pack-years (0.002), had more involved sites $(p=0.011)$, more prior therapies $(p=0.001)$, and a lower mean albumin level $(p=0.014)$ (Table 2). The two groups did not differ significantly with respect to any of the other baseline characteristics, although there was a trend toward higher KPS scores $(p=0.086)$ in the resistant group. We additionally found no consistent effects of line of therapy, particular drug or drug class on initial clinical benefit for anti-PD1/L1 treatment. All patients with EGFR or ALK mutation had received prior TKI per standard of care before receiving anti-PD1/L1. Chemotherapy regimens varied but were predominately based on platinum chemotherapy. Relative to radiation, we also found no impact on progressionfree or overall survival for radiation either prior to immunotherapy or radiation at any point in the patients treatment course.

\section{Acquired resistance characteristics}

Of the fifty seven patients with initial clinical benefit, thirty four $(59.6 \%)$ subsequently progressed $(n=33)$ or died absent a prior progression $(n=1)$. To assess factors associated with acquired resistance, we evaluated PFS and OS in the subgroup of patients who had initial clinical benefit. For this analysis, time was 
Table 1: Baseline clinical and demographic characteristics

\begin{tabular}{|c|c|c|}
\hline Characteristic & No $(n=93)$ & $\%$ \\
\hline Age, Mean (range) & $69.1(48-87)$ & - \\
\hline \multicolumn{3}{|l|}{ Sex } \\
\hline Male & 48 & $51.6 \%$ \\
\hline Female & 45 & $48.4 \%$ \\
\hline \multicolumn{3}{|l|}{ Smoking History } \\
\hline Yes & 86 & $92.5 \%$ \\
\hline No & 7 & $7.5 \%$ \\
\hline Pack-years, Mean (range) ${ }^{\mathrm{a}}$ & 35.7 & $(0-100)$ \\
\hline \multicolumn{3}{|l|}{ Histology } \\
\hline Adenocarcinoma & 63 & $67.7 \%$ \\
\hline Squamous & 28 & $30.1 \%$ \\
\hline Non-small cell carcinoma & 2 & $2.2 \%$ \\
\hline \multicolumn{3}{|l|}{ Stage } \\
\hline M1a & 32 & $34.4 \%$ \\
\hline M1b & 61 & $65.6 \%$ \\
\hline \multicolumn{3}{|l|}{ KPS $^{\mathbf{b}}$} \\
\hline 0 & 12 & $13.0 \%$ \\
\hline 1 & 54 & $58.7 \%$ \\
\hline 2 & 23 & $25.0 \%$ \\
\hline 3 & 3 & $3.3 \%$ \\
\hline Brain metastases (Treated) & 13 & $14.0 \%$ \\
\hline \multicolumn{3}{|c|}{ Number of sites with at least one lesion } \\
\hline 1 & 26 & $28.0 \%$ \\
\hline 2 & 40 & $43.0 \%$ \\
\hline 3 & 19 & $20.4 \%$ \\
\hline 4 & 6 & $6.4 \%$ \\
\hline 5 & 2 & $2.2 \%$ \\
\hline \multicolumn{3}{|l|}{ Mutational status ${ }^{\mathrm{c}}$} \\
\hline KRAS & 20 & $33.3 \%$ \\
\hline EGFR & 6 & $10.0 \%$ \\
\hline ALK & 1 & $1.7 \%$ \\
\hline WT & 33 & $55.0 \%$ \\
\hline \multicolumn{3}{|l|}{ Number of prior therapies } \\
\hline 3 & 14 & $15.0 \%$ \\
\hline 2 & 37 & $39.8 \%$ \\
\hline 1 & 41 & $44.1 \%$ \\
\hline 0 & 1 & $1.1 \%$ \\
\hline \multicolumn{3}{|l|}{ Immunotherapy Agent } \\
\hline Nivolumab & 89 & $95.7 \%$ \\
\hline Pembrolizumab & 2 & $2.2 \%$ \\
\hline atezolizumab & 2 & $2.2 \%$ \\
\hline \multicolumn{3}{|l|}{ Best Prior Response $^{b}$} \\
\hline $\mathrm{CR}$ & 6 & $6.5 \%$ \\
\hline PR & 31 & $33.7 \%$ \\
\hline SD & 26 & $28.3 \%$ \\
\hline PD & 29 & $31.5 \%$ \\
\hline Albumin, Mean (range) ${ }^{d}$ & $3.4(2.0-4.4$ & \\
\hline
\end{tabular}

${ }^{\mathrm{a}} 6$ missing; ${ }^{\mathrm{b}} 1$ missing; ${ }^{\mathrm{c}} 33$ missing; ${ }^{\mathrm{d}} 18$ missing. 
Table 2: Comparison of baseline characteristics in patients with primary resistance vs. Initial benefit

\begin{tabular}{|c|c|c|c|}
\hline Characteristic & $\begin{array}{c}\text { Primary resistance } \\
(n=36)\end{array}$ & $\begin{array}{c}\text { Initial benefit } \\
\quad(n=57)\end{array}$ & $P$-value \\
\hline Age, Mean + SE & $68.4 \pm 1.6$ & $69.6 \pm 1.2$ & 0.53 \\
\hline \multicolumn{4}{|l|}{ Sex } \\
\hline Male & $17(47.2 \%)$ & $28(49.1 \%)$ & \multirow[t]{2}{*}{1.0} \\
\hline Female & $19(52.8 \%)$ & $29(50.9 \%)$ & \\
\hline \multicolumn{4}{|l|}{ Smoking History } \\
\hline Yes & $30(83.3 \%)$ & $56(98.2 \%)$ & \multirow[t]{2}{*}{0.013} \\
\hline No & $6(16.7 \%)$ & $1(1.8 \%)$ & \\
\hline Pack years, Mean + SE $^{a}$ & $26.7 \pm 3.9$ & $41.8 \pm 2.8$ & 0.002 \\
\hline \multicolumn{4}{|l|}{ Histology } \\
\hline Adenocarcinoma & $27(75.0 \%)$ & $36(63.2 \%)$ & \multirow[t]{3}{*}{0.40} \\
\hline Squamous & $0(0.0 \%)$ & $2(3.5 \%)$ & \\
\hline Non-small cell carcinoma & $9(25.0 \%)$ & $19(33.3 \%)$ & \\
\hline \multicolumn{4}{|l|}{ Stage } \\
\hline M1a & $9(25.0 \%)$ & $23(40.4 \%)$ & \multirow[t]{2}{*}{0.18} \\
\hline M1b & $27(75.0 \%)$ & $34(59.6 \%)$ & \\
\hline \multicolumn{4}{|l|}{ KPS $^{\mathbf{b}}$} \\
\hline 0 & $3(8.3 \%)$ & $9(16.1 \%)$ & \multirow[t]{4}{*}{0.086} \\
\hline 1 & $18(50.0 \%)$ & $36(64.3 \%)$ & \\
\hline 2 & $14(38.9 \%)$ & $9(16.1 \%)$ & \\
\hline 3 & $1(2.8 \%)$ & $2(3.6 \%)$ & \\
\hline Brain metastases (Treated) & $5(13.9 \%)$ & $8(14.0 \%)$ & 1.0 \\
\hline \multicolumn{4}{|c|}{ Number of sites with at least one lesion } \\
\hline 1 & $5(13.9 \%)$ & $21(36.8 \%)$ & \multirow[t]{5}{*}{0.011} \\
\hline 2 & $16(44.4 \%)$ & $24(42.1 \%)$ & \\
\hline 3 & $8(22.2 \%)$ & $11(19.3 \%)$ & \\
\hline 4 & $5(13.9 \%)$ & $1(1.8 \%)$ & \\
\hline 5 & $2(5.6)$ & $0(0.0 \%)$ & \\
\hline \multicolumn{4}{|l|}{ Mutational status } \\
\hline KRAS & $0(0.0 \%)$ & $1(2.8 \%)$ & \multirow[t]{4}{*}{0.59} \\
\hline EGFR & $3(12.5 \%)$ & $3(8.3 \%)$ & \\
\hline ALK & $6(25.0 \%)$ & $14(38.9 \%)$ & \\
\hline WT & $15(62.5 \%)$ & $18(50.0 \%)$ & \\
\hline \multicolumn{4}{|l|}{ Number of prior therapies } \\
\hline 3 & $12(33.3 \%)$ & $2(3.5 \%)$ & \multirow[t]{4}{*}{0.001} \\
\hline 2 & $12(33.3 \%$ & $25(43.9 \%)$ & \\
\hline 1 & $12(33.3 \%)$ & $29(50.9 \%)$ & \\
\hline 0 & $0(0.0 \%)$ & $1(1.8 \%)$ & \\
\hline \multicolumn{4}{|l|}{ Immunotherapy Agent } \\
\hline Nivolumab & $0(0.0 \%)$ & $2(3.5 \%)$ & \multirow[t]{3}{*}{0.77} \\
\hline Pembrolizumab & $35(97.2 \%)$ & $54(94.7 \%)$ & \\
\hline atezolizumab & $1(2.8 \%)$ & $1(1.8 \%)$ & \\
\hline \multicolumn{4}{|l|}{ Best Prior Response $^{b}$} \\
\hline $\mathrm{CR}$ & $2(5.6 \%)$ & $4(7.1 \%)$ & \multirow[t]{4}{*}{0.33} \\
\hline PR & $11(30.6 \%)$ & $20(35.7 \%)$ & \\
\hline SD & $14(38.9 \%)$ & $12(21.4 \%)$ & \\
\hline PD & $9(25.0 \%)$ & $20(35.7 \%)$ & \\
\hline Albumin, Mean (range) ${ }^{d}$ & $3.2 \pm 0.12$ & $3.5 \pm 0.07$ & 0.014 \\
\hline
\end{tabular}

a6 missing; ${ }^{\mathrm{b}} 1$ missing; c33 missing; ${ }^{\mathrm{d}} 18$ missing. 
measured from the date of the initial CT evaluation and depth of tumor response to the anti-PD-1 agent was included among the predictor variables. Figure 1 shows the Kaplan-Meier curves. Supplementary Table 1, presents the results of fitting univariate Cox regression models for PFS and OS. Factors significantly associated with progression-free survival were $\operatorname{KPS}(p=0.004)$ and depth of response to the anti-PD-1 therapy $(p=0.003)$. KPS was also significantly associated with overall survival $(p=0.010)$ and depth of response was marginally significant $(p=0.053)$. Molecular status specifically for KRAS mutation was tested and did not show a significant association with progression-free survival. Other mutations such as EGFR and ALK were not present in enough samples to support an analysis in these sub-populations. Multivariable analyses were then fit including univariate predictors significant at $p<0.15$, followed by backward elimination until only statistical significant predictors remained. (Mutational status was omitted from the multivariable analyses due to a high rate of missing data (see Supplementary Table 2). KPS and depth of response remained in the final model for PFS; histology, number of involved sites, and depth of response were included in the final model for OS. PFS and OS curves for patients with a $30 \%$ or greater reduction in tumor size following anti-PD-1/PD-L1 treatment compared to those with less shrinkage are shown in Figure 2.
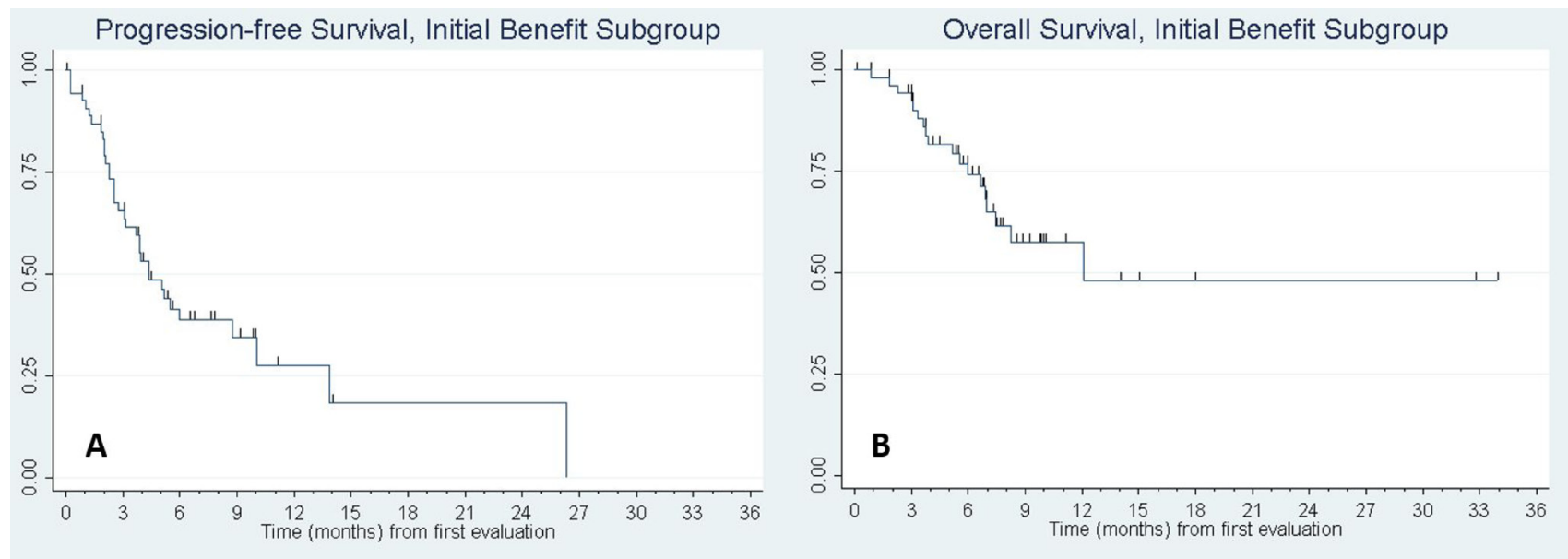

Figure 1: Kaplan-Meier curve of (A) progression-free survival and (B) overall survival of patients with initial benefit. $N=57$ patients had initial benefit to anti-PD-1/PD-L1 monotherapy. Of these patients, median PFS was 4.4 months, 95\% CI: (3.1, 8.7). Median OS was 12.1 months, $95 \%$ CI: (7.0, -). Tic marks denote censored observations.
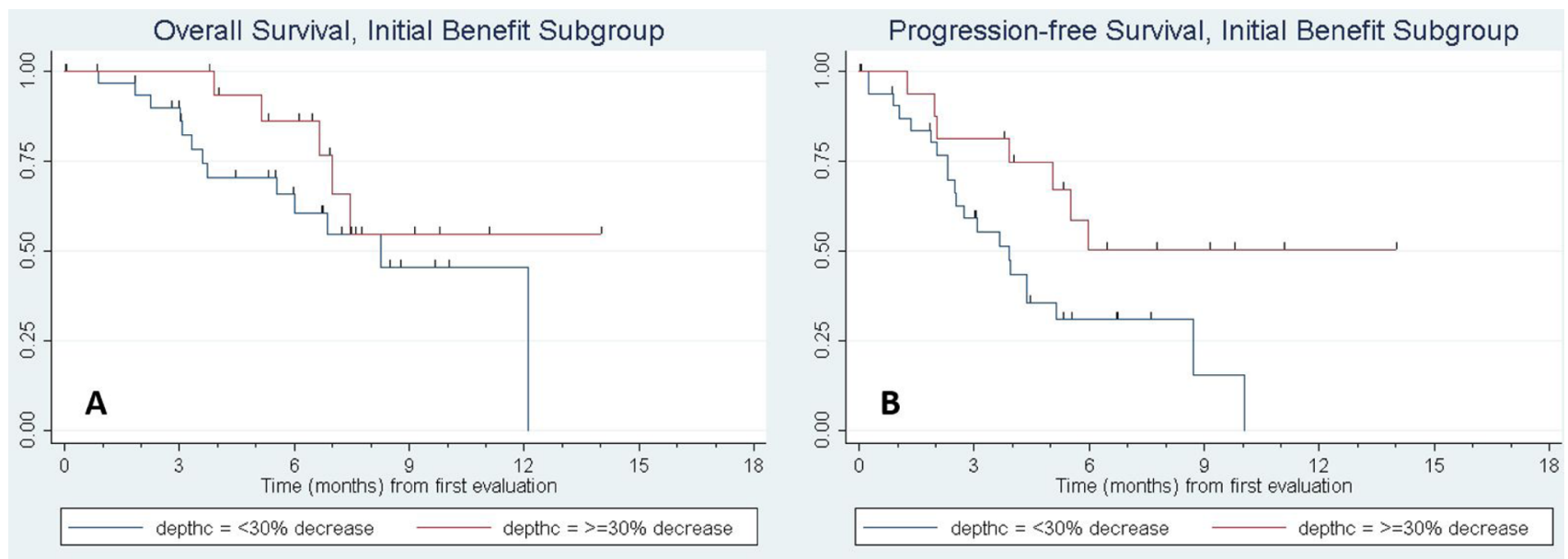

Figure 2: Kaplan-Meier curve of (A) progression-free survival and (B) overall survival of patients with initial benefit based on depth of response. The red curve denotes patients with $\geq 30 \%$ response in tumor size by RECIST criteria to anti-PD-1/PD-L1 therapy, while the blue line denotes response $<30 \%$. On multivariate cox regression analysis, PFS and OS were significantly longer for patients with increasing depth of response, with a hazard ratio (HR) of $0.74,95 \% \mathrm{CI}(0.62,0.89), p=0.002$ for PFS and HR of $0.79(0.65,0.93), p=0.006$ for OS per every $10 \%$ increase in depth of response. 


\section{Characteristics of progression and post- progression course}

In patients with acquired resistance, the majority of patients $(n=20,60.6 \%)$ had progression of existing disease rather than the development of new disease. Twenty two $(66.7 \%)$ of the patients developed isolated (single organ) sites of progression, while $n=10$ had more diffuse, systemic progression. All patients were receiving anti-PD-1 therapy at time of progression. Seventeen patients $(51.5 \%)$ received further systemic therapy and 2 patients $(6.1 \%)$ received local radiation therapy. The majority of these patients $(n=27,81.8 \%)$ had progressive disease as best response to further systemic therapy. Four patients $(12.1 \%)$ had a partial response. Local therapy was rare. The two patients that received radiation therapy have continued on active surveillance, and have not yet required systemic therapy after 9 months of surveillance.

\section{Immune gene expression profiling}

Through immune expression profiling of 1016 TCGA samples, three distinct tumor subtypes with low, moderate, and high expression level of the T-cell genes emerged. (Figure 3). Overall, the presence of the "T cellinflamed" microenvironment was identified at a higher percentage in adenocarcinoma (42\%) than squamous cell carcinoma patients $(21 \%)$.

The proportion of adenocarcinoma tumor samples were further categorized by mutational subtype (KRAS, EGFR, ALK, or ROS1) and the proportion of "T cellinflamed" versus "non-inflamed" was analyzed, as well as expression level of non-synonymous somatic mutations (NSSMs) - an indicator of mutational loadand PD-L1 expression (Figure 4). EGFR mutated sample demonstrated a higher proportion of "T cell-inflamed" tumors versus "non-inflamed" at marginal significance ( $17 \%$ versus $7 \%$, respectively, $p=0.047)$. Otherwise, no significant differences were noted based on mutational subtype. There was no significant difference in expression of PD-L1 based on driver mutation, though ALK and ROS1 were not analyzed as they had too few samples. While on average, the mutational load was slightly lower in "inflamed" tumors relative to "non-inflamed" tumors in adenocarcinoma samples, further analysis of neoantigen load demonstrated no difference between "inflamed" versus "non-inflamed" subgroups in any of the driver mutation samples (see Supplementary Figure 1). Furthermore, data in melanoma has demonstrated no correlation between immune gene expression and either mutational load or neoantigen load [11].

\section{DISCUSSION}

In this analysis of clinical and molecular factors influencing resistance to anti-PD1/L1 therapy in NSCLC we observed no clinical differences in innate resistance however noted multiple clinical factors associated with acquired resistance and additionally noted no differences in neoantigen load between $\mathrm{T}$ cell-inflamed and non- $\mathrm{T}$ cell-inflamed NSCLC tumors. In patients who developed acquired resistance, the median time to subsequent disease progression or death was 4.4 months beyond the time of their first CT evaluation. Factors associated with improved PFS in these patients were lower KPS score and depth of response to the anti-PD- 1 therapy $>30 \%$. Factors associated with subsequent survival were adenocarcinoma histology, number of involved sites, and depth of response $>30 \%$.

Relevant to clinical practice, resistance was most often characterized by progression of an existing (rather than new) lesions. This progression was often isolated to a single site, rather than diffuse progression. Despite this confined progression, at our institutions local therapy appeared to be underutilized, with only 2 patient having radiation therapy after developing resistance. Pseudoprogression is a phenomenon first described in melanoma clinical trials suggesting that up to $15 \%$
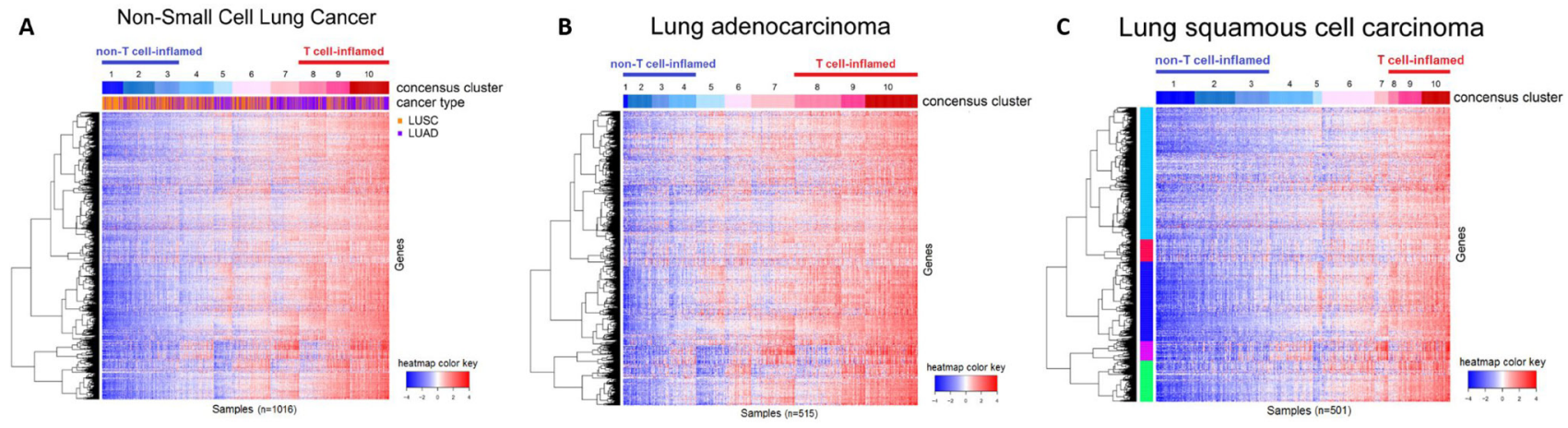

Figure 3: Immune gene expression heatmap of (A) all NSCLC samples, (B) lung adenocarcinoma (LUAC) samples, (C) lung squamous cell carcinoma (LUSC) samples. "T cell-inflamed" and "T cell-non-inflamed" tumors can be distinguished by immune gene expression profiling. In LUAC samples, $80(15.53 \%)$ of the tumors showed minimal expression of T-cell-related immune genes ("T cell-noninflamed"), whereas 215 (41.75\%) show over-expression ("T cell-inflamed"). In LUSC samples, 193 (38.52\%) showed minimal expression ("T cell-non-inflamed") and 105 (20.96\%) showed high expression ("T cell-inflamed"). 
of patients may manifest RECIST progressive disease prior to showing clinical benefit [12]. In NSCLC this phenomenon is much more rarely observed [13]. Clinical trials integrating multi-modal management of isolated sites of recurrence in NSCLC may be indicated to expand the benefit of PD1/L1 based immunotherapy.

From our cohort of NSCLC patients, little or no archival tissue was available due to prior clinical trial participation or standard diagnostic testing and we therefore turned to TCGA for interrogation of NSCLC at the molecular level. As TCGA captures patients prior to systemic therapy, we hypothesized that this sample set may be consistent on a molecular level with our analysis of pre-treatment factors associated with treatment resistance. In the NSCLC cohort of the TCGA database, a "T cell-inflamed" tumor microenvironment was more common in lung adenocarcinomas (42\%), versus lung squamous cell carcinomas $(21 \%)$. This will need to be investigated further in clinical trials, as current clinical data does not support a significantly more robust response in lung adenocarcinoma histologies. The TCGA analysis suggested that no molecular driver mutations are significantly associated with a "T cell-inflamed" tumor microenvironment, though data on EGFR mutated samples suggested a marginally significant increase in "T cell-inflamed" tumors (7\% in "non-inflamed" versus $17 \%$ in "inflamed"). While similar signatures are highly associated with response to anti-PD1/L1 Ab, they are not perfectly predictive $[7,14]$. The presence of the T cell-inflamed microenvironment in these EGFR mutant samples suggests that pure $\mathrm{T}$ cell-exclusion is unlikely to be a major mechanism of resistance. Alternative hypotheses could be resistance driven by increased presence of interferon- $\gamma$ associated molecules such as secondary immune checkpoints including TIM3 [15] and/or Treg cells or alternatively activation of other immunosuppressive pathways such as CD73 [16, 17], among other possibilities.

The significant proportion of patients with initial clinical benefit in this study $(61.3 \%)$ is significantly higher than the $19-20 \%$ response rates seen in large phase III trials investigating checkpoint inhibitors. While the reason for this is unclear, it is observed that patients in this retrospective study appeared to have less volume disease
A

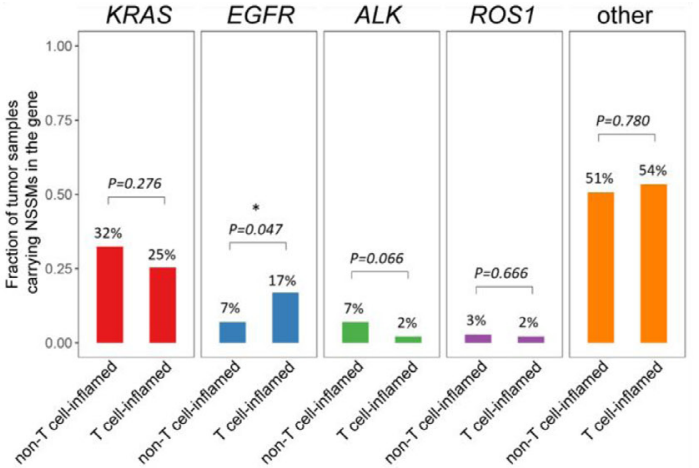

B

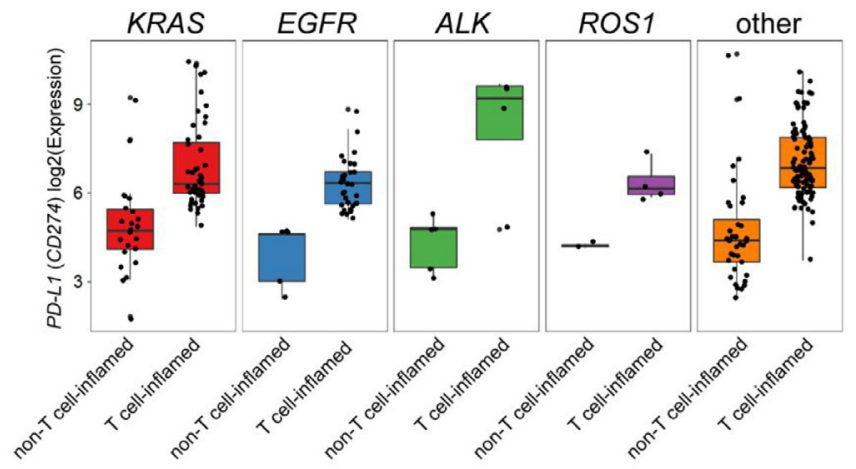

C

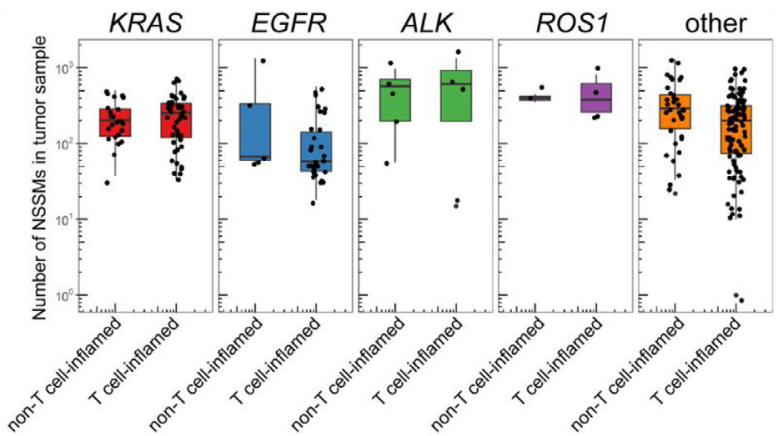

Figure 4: Analysis of lung adenocarcinoma samples based on molecular driver mutation (ALK, EGFR, KRAS, ROS1, or wild-type (none)). (A) "T cell-inflamed" versus "T cell-non-inflamed" samples, (B) PD-L1 (CD-47) expression, and (C) number of neoantigens were all evaluated. No significant differences in "T cell-inflamed" versus "T cell-non-inflamed" was observed based on molecular driver mutation, though a higher concentration of "T cell-inflamed" samples were detected in the EGFR samples that bordered on significance $(17 \%$ versus $7 \%, p=0.047)$. Similar level of PD-L1 expression and neoantigens were present regardless of mutation. Only mutually exclusive samples were included, samples with mutations in more than one driver mutation $(n=15)$ were not included. ${ }^{*}$ NSSMs $=$ nonsynonymous somatic mutations (missense, nonsense, splice, insertions/deletions). Number on top of each bar represents the percentage of tumor samples carrying NSSM in each of the driver genes within non-T cell-inflamed or T cell-inflamed tumor group, which corresponds to the $\mathrm{y}$-axis but in percentage units. 
when initiating therapy (suggested by $91 \%$ of our patients having 3 sites of disease or less) compared to the subjects in these phase III trials.

This study describes key clinical criteria that may be predictive of innate resistance, acquired resistance, or death with anti-PD-1/PD-L1 therapy. Further investigations to confirm this data and better identify these patients is critical to help with treatment algorithms for this aggressive disease. The molecular analysis of the TCGA database suggests that lung adenocarcinoma tumors may be more commonly associated with a " $T$ cell-inflamed" microenvironment, and thus possibly more likely to respond to immunotherapy. However, as of yet this has not been noted in larger, phase III clinical trials.

\section{MATERIALS AND METHODS}

\section{Patients}

Following approval from each site's institutional review board (IRB), we screened all patients diagnosed with stage IV NSCLC who received single-agent antiPD-1/PD-L-1 therapy (nivolumab, pembrolizumab, or atezolizumab). The sites included the University of Chicago Cancer Center $(n=37)$, NorthShore University HealthSystems $(n=50)$, and Ingalls HealthSystems $(n=6)$. As the data were retrospective, waiver of consent was obtained at all sites. We included patients with both squamous and non-squamous histology who had received at least one dose of anti-PD-1 therapy. The schedule for radiographic tumor assessment for each patient was physician's choice, though typically CT scans were performed every 8 weeks unless clinically indicated. The start date of anti-PD-1/PD-L1 therapy for the first patient was $4 / 6 / 15$ and data were last reviewed on 12/15/16.

\section{Study design}

We obtained baseline demographic data for each patient including age, gender, American Joint Committee on Cancer (AJCC) pathologic stage, and performance status defined by the Eastern Cooperative Oncology Group (ECOG). Additional information regarding prior treatments and responses were also recorded. To assess the efficacy of initial anti-PD-1 therapy, we evaluated the objective response based on RECIST v1.1 criteria performed by the investigator, progression free survival (PFS), and overall survival (OS). Finally, to characterize the post-progression data, we collected data following disease progression including sites of progression, subsequent treatments, responses, and survival. Innate resistance was defined as disease progression by RECIST criteria on first $\mathrm{CT}$ evaluation or death prior to first $\mathrm{CT}$ evaluation; initial benefit was defined as alive with stable disease, partial, or complete response by RECIST criteria at first CT scan. Stable disease was included with response based on the hypothesis that the tumor microenvironment in patients with initial stable disease would be more similar to patients with initial response than those patients demonstrating initial progression. While this is an area of active translational research multiple clinical trials are ongoing using this model $[18,19]$.

\section{Statistical analysis}

Descriptive statistics (means, standard deviations, and percentages) were generated for baseline clinical and demographic data. OS and PFS were calculated based on the Kaplan-Meier method [20]. PFS was defined as time from the start of treatment until disease progression or death from any cause. Overall survival was defined as time from the start of treatment until death for any reason. Patients were censored at their last follow-up if no event had occurred. Ninety-five percent confidence intervals for the medians were derived using the method of Brookmeyer and Crowley [21]. Subsequent analyses were performed in two parts. First, we compared the baseline characteristics between patients with innate resistance, defined as disease progression on first $\mathrm{CT}$ evaluation or death prior to first $\mathrm{CT}$ evaluation, and patients with initial benefit, i.e., alive with stable disease, partial, or complete response at first CT scan. These comparisons consisted of two-sample $t$-tests for continuous variables and chi-square or Fisher's exact test for discrete data. Second, among the subset of patients with initial benefit, we constructed KaplanMeier curves and fitted univariate and multivariable Cox regression models [22] for PFS and OS to identify factors predictive of time to disease progression or death. Here, PFS and OS were measured from the time of the first CT scan, and candidate predictor variables included the initial depth of response to the treatment (percentage change in tumor size from baseline to first CT evaluation calculated on a continuous scale). In patients with initial benefit who subsequently progressed, their post-progression survival times were summarized using the Kaplan-Meier estimator.

\section{Analysis of TCGA data set}

The Cancer Genome Atlas NSCLC level 4 RNA-seq gene expression data and level 2 somatic mutation data were downloaded from Broad Genome Data Analysis Center (http://gdac.broadinstitute.org) (release date 01/28/2016). A total of 515 adenocarcinoma (LUAD) and 501 squamous cell carcinoma (LUSC) primary tumors were analyzed. RNA-seq raw read counts were summarized at the gene level using the RSEM (RNA-Seq by Expectation Maximization) method, and were upper quartile-normalized and $\log _{2}$-transformed across all 1,016 samples in this study.

T-cell-inflamed and non-T-cell-inflamed group's tumor groups were identified by our previously described method [23]. In brief, unsupervised hierarchical clustering 
with $\mathrm{K}$ equal to 10 was performed on 17,867 genes that are expressed in at least $50 \%$ of the samples. A cluster of 943 genes including 12 genes of a previously described T-cell gene signature (CD8A, CCL3, CCL4, CXCL9, CXCL10, ICOS, IRF1, GZMK, HLA-DMA, HLADMB, HLA-DOA, and HLA-DOB) were selected for the identification of $\mathrm{T}$ cell-inflamed, intermediate, and non- $\mathrm{T}$ cell-inflamed tumor groups using a consensus clustering algorithm with 2,000 resampling cycles, unsupervised hierarchical clustering method and Euclidean distance [24] (ConsensusClusterPlus, v1.38.0). Whole exome somatic variants were filtered to retrieve NSSMs (nonsynonymous somatic mutations) defined as missense mutations, nonsense mutations, small insertions/deletions, and those affecting splicing site of a protein-coding transcript. Predicted neoantigen data were obtained from a previous published study [25].

\section{CONFLICTS OF INTEREST}

Authors declare no conflicts of interest.

\section{REFERENCES}

1. Howlader N, Noone AM, Krapcho M, Miller D, Bishop K, Altekruse SF, Kosary CL, Yu M, Ruhl J, Tatalovich Z, Mariotto A, Lewis DR, Chen HS, et al, eds. SEER Cancer Statistics Review. 1975-2013, National Cancer Institute. Bethesda, MD, based on November 2015 SEER data submission, posted to the SEER web site, April 2016. http:// seer.cancer.gov/csr/1975_2013/.

2. Borghaei H, Paz-Ares L, Horn L, Spigel DR, Steins M, Ready NE, Chow LQ, Vokes EE, Felip E, Holgado E, Barlesi F, Kohlhäufl M, Arrieta O, et al. Nivolumab versus Docetaxel in Advanced Nonsquamous Non-Small-Cell Lung Cancer. N Engl J Med. 2015; 373:1627-39. https:// doi.org/10.1056/NEJMoa1507643.

3. Brahmer J, Reckamp KL, Baas P, Crinò L, Eberhardt WE, Poddubskaya E, Antonia S, Pluzanski A, Vokes EE, Holgado E, Waterhouse D, Ready N, Gainor J, et al. Nivolumab versus Docetaxel in Advanced SquamousCell Non-Small-Cell Lung Cancer. N Engl J Med. 2015; 373:123-35. https://doi.org/10.1056/NEJMoa1504627.

4. Reck M, Rodríguez-Abreu D, Robinson AG, Hui R, Csőszi T, Fülöp A, Gottfried M, Peled N, Tafreshi A, Cuffe S, O'Brien M, Rao S, Hotta K, et al, and KEYNOTE-024 Investigators. Pembrolizumab versus Chemotherapy for PD-L1-Positive Non-Small-Cell Lung Cancer. N Engl J Med. 2016; 375:1823-33. https://doi. org/10.1056/NEJMoa1606774.

5. Langer CJ, Gadgeel SM, Borghaei H, Papadimitrakopoulou VA, Patnaik A, Powell SF, Gentzler RD, Martins RG, Stevenson JP, Jalal SI, Panwalkar A, Yang JC, Gubens M, et al, and KEYNOTE-021 investigators. Carboplatin and pemetrexed with or without pembrolizumab for advanced, non-squamous non-small-cell lung cancer: a randomised, phase 2 cohort of the open-label KEYNOTE-021 study. Lancet Oncol. 2016; 17:1497-508. https://doi.org/10.1016/ S1470-2045(16)30498-3.

6. Taube JM, Klein A, Brahmer JR, Xu H, Pan X, Kim JH, Chen L, Pardoll DM, Topalian SL, Anders RA. Association of PD-1, PD-1 ligands, and other features of the tumor immune microenvironment with response to anti-PD-1 therapy. Clin Cancer Res. 2014; 20:5064-74. https://doi. org/10.1158/1078-0432.CCR-13-3271.

7. Herbst RS, Soria JC, Kowanetz M, Fine GD, Hamid O, Gordon MS, Sosman JA, McDermott DF, Powderly JD, Gettinger SN, Kohrt HE, Horn L, Lawrence DP, et al. Predictive correlates of response to the anti-PD-L1 antibody MPDL3280A in cancer patients. Nature. 2014; 515:563-67. https://doi.org/10.1038/nature14011.

8. Harlin H, Meng Y, Peterson AC, Zha Y, Tretiakova M, Slingluff C, McKee M, Gajewski TF. Chemokine expression in melanoma metastases associated with CD8+ T-cell recruitment. Cancer Res. 2009; 69:3077-85. https:// doi.org/10.1158/0008-5472.CAN-08-2281.

9. Ribas A, Robert C, Hodi FS, Wolchok JD, Joshua AM, Hwu WJ, Weber JS, Zarour HM, Kefford R, Loboda A, Albright A, Kang S, Ebbinghaus S, et al. Association of response to programmed death receptor 1 (PD-1) blockade with pembrolizumab (MK-3475) with an interferon inflammatory immune gene signature. J Clin Oncol. 2015; 33:abstr 3001.

10. Ji RR, Chasalow SD, Wang L, Hamid O, Schmidt H, Cogswell J, Alaparthy S, Berman D, Jure-Kunkel M, Siemers NO, Jackson JR, Shahabi V. An immune-active tumor microenvironment favors clinical response to ipilimumab. Cancer Immunol Immunother. 2012; 61:101931. https://doi.org/10.1007/s00262-011-1172-6.

11. Spranger S, Luke JJ, Bao R, Zha Y, Hernandez KM, Li Y, Gajewski AP, Andrade J, Gajewski TF. Density of immunogenic antigens does not explain the presence or absence of the T-cell-inflamed tumor microenvironment in melanoma. Proc Natl Acad Sci USA. 2016; 113:E7759-68. https://doi.org/10.1073/pnas.1609376113.

12. Wolchok JD, Hoos A, O'Day S, Weber JS, Hamid O, Lebbé C, Maio M, Binder M, Bohnsack O, Nichol G, Humphrey R, Hodi FS. Guidelines for the evaluation of immune therapy activity in solid tumors: immune-related response criteria. Clin Cancer Res. 2009; 15:7412-20. https://doi.org/10.1158/1078-0432.CCR-09-1624.

13. Nishino M, Dahlberg SE, Adeni AE, Lydon CA, Hatabu H, Jänne PA, Hodi FS, Awad MM. Tumor response dynamics of advanced non-small cell lung cancer patients treated with PD-1 inhibitors: imaging markers for treatment outcome. Clin Cancer Res. 2017; 23:5737-44. https://doi. org/10.1158/1078-0432.CCR-17-1434.

14. Ayers M, Lunceford J, Nebozhyn M, Murphy E, Loboda A, Kaufman DR, Albright A, Cheng JD, Kang SP, Shankaran 
V, Piha-Paul SA, Yearley J, Seiwert TY, et al. IFN- $\gamma$-related mRNA profile predicts clinical response to PD-1 blockade. J Clin Invest. 2017; 127:2930-40. https://doi.org/10.1172/ JCI91190.

15. Koyama S, Akbay EA, Li YY, Herter-Sprie GS, Buczkowski KA, Richards WG, Gandhi L, Redig AJ, Rodig SJ, Asahina H, Jones RE, Kulkarni MM, Kuraguchi M, et al. Adaptive resistance to therapeutic PD-1 blockade is associated with upregulation of alternative immune checkpoints. Nat Commun. 2016; 7:10501. https:/doi. org/10.1038/ncomms10501.

16. Streicher K, Higgs BW, Wu S, Coffman K, Damera G, Durham N, Greenlees L, Lazdun Y, Cheng L, Cooper Z. Ranade, K. Increased CD73 and reduced IFNG signature expression in relation to response rates to anti-PD-1(L1) therapies in EGFR-mutant NSCLC. J Clin Oncol. 2017; 35:11505-11505.

17. Inoue $\mathrm{Y}$, Yoshimura $\mathrm{K}$, Kurabe $\mathrm{N}$, Kahyo T, Kawase A, Tanahashi M, Ogawa H, Inui N, Funai K, Shinmura K, Niwa H, Suda T, Sugimura H. Prognostic impact of CD73 and $\mathrm{A} 2 \mathrm{~A}$ adenosine receptor expression in non-small-cell lung cancer. Oncotarget. 2017; 8:8738-51. https://doi. org/10.18632/oncotarget.14434.

18. Fong L, Forde PM, Powderly JD, Goldman JW, Nemunaitis JJ, Luke JJ, Hellmann MD, Kummar S, Doebele RC, Mahadevan D, Gadgeel SM, Hughes B, Markman B. Safety and clinical activity of adenosine A2a receptor (A2aR) antagonist, CPI-444, in anti-PD1/PDL1 treatment-refractory renal cell (RCC) and non-small cell lung cancer (NSCLC) patients. J Clin Oncol. 2017; 35:3004-3004.

19. Ascierto PA, Melero I, Bhatia S, Bono P, Sanborn RE, Lipson EJ, Callahan MK, Gajewski T, Gomez-Roca CA, Hodi FS, Curigliano G, Nyakas M, Preusser M. Initial efficacy of anti-lymphocyte activation gene-3 (anti-LAG-3; BMS-986016) in combination with nivolumab (nivo) in pts with melanoma (MEL) previously treated with anti-PD-1/ PD-L1 therapy. J Clin Oncol. 2017; 35:9520-9520.

20. Kaplan EL, Meier P. Nonparametric estimation from incomplete observations. J Am Stat Assoc. 1958; 53:45781. https://doi.org/10.1080/01621459.1958.10501452.

21. Brookmeyer R, Crowley JJ. A confidence interval for the median survival time. Biometrics. 1982; 38:29-41. https:// doi.org/10.2307/2530286.

22. Cox DR. Regression models and life tables (with discussion). J R Stat Soc B. 1972; 34:187-220.

23. Spranger S, Bao R, Gajewski TF. Melanoma-intrinsic $\beta$-catenin signalling prevents anti-tumour immunity. Nature. 2015; 523:231-35. https://doi.org/10.1038/nature14404.

24. Wilkerson MD, Hayes DN. ConsensusClusterPlus: a class discovery tool with confidence assessments and item tracking. Bioinformatics. 2010; 26:1572-73. https://doi. org/10.1093/bioinformatics/btq170.

25. Rooney MS, Shukla SA, Wu CJ, Getz G, Hacohen N. Molecular and genetic properties of tumors associated with local immune cytolytic activity. Cell. 2015; 160:48-61. https://doi.org/10.1016/j.cell.2014.12.033. 